\title{
Bioinspired-Interpenetrating Network (IPNs) Hydrogel (BIOF-INPs) and TMD in Vitro: Bioadhesion, Drug Release and Build in Free Radical Detection and Defense
}

\author{
Victoria Tamara Perchyonok ${ }^{1}$, Vanessa Reher ${ }^{2}$, Nicolaas J. Basson ${ }^{3}$, Shengmiao Zhang4, \\ Sias R. Grobler ${ }^{3}$ \\ ${ }^{1}$ VTPCHEM PTY LTD, Gold Coast, Australia \\ ${ }^{2}$ School of Dentistry and Oral Health, Griffith University, Gold Coast, Australia \\ ${ }^{3}$ Oral and Dental Research Institute, Faculty of Dentistry, University of the Western Cape, Cape Town, \\ South Africa \\ ${ }^{4}$ School of Material Science and Engineering, East China University of Science and Technology, Shanghai, China \\ Email: tamaraperchyonok@gmail.com
}

Received 11 January 2015; accepted 27 February 2015; published 4 March 2015

Copyright (C) 2015 by authors and Scientific Research Publishing Inc.

This work is licensed under the Creative Commons Attribution International License (CC BY).

http://creativecommons.org/licenses/by/4.0/

(c) (i) Open Access

\begin{abstract}
In this work, Bioactive-functionalized interpenetrating network (IPNs) hydrogel (BIOF-INPs) were prepared and investigated in vitro for the free radical detection/defense, therapeutic release as well as shear bond strength to dentine, ability to re-mineralize surface of the dentin after application of these bio-inspired materials using a biologically inspired mineralization process in vitro as well as investigating antimicrobial properties of the BIOF-INPs against $S$. aureous. The aim of this investigation was to evaluate the suitability and flexibility of the designer materials to act as an "in vitro" probe to gain insights into molecular origin of TMD and associated disorders.
\end{abstract}

\section{Keywords}

TMD, Functionalized IPNs, Bio-Adhesion, Drug Release, Build in Free Radical Detection and Defense

\section{Introduction}

In the last few decades, tissue engineering has emerged as a promising multidisciplinary approach for the repair 
and regeneration of damaged bone tissue [1] [2].

The molecular events that underline the degenerative temporomandibular joint diseases are poorly understood [3].

Mechanical stresses are generated during functional or para-functional movements of the jaw; adaptive mechanism of the TMJ may be exceeded by free radical accumulation leading to a dysfunctional state (i.e. disease state) [4]. Biologically relevant free radicals are very reactive and unstable molecular entities that have an unpaired electron and they can produce participate in the propagation chain reaction to form a new radical. Although oxygen free radicals participate in many physiological processes, they can be harmful to tissue when either their action or their generation have been left uncontrolled. The most common source of free radicals in biological systems is oxygen. The elevation of reactive oxygen species (ROS) leads to oxidative stress that causes molecular damage to the vital structures and functions [5]-[7].

The unsaturated fatty acids of cell membrane lipid are susceptible to peroxidative reaction. Lipid peroxidation of cell membranes has been implicated in the wide range of tissue injuries and diseases. Accumulation of lipid hydroperoxides in a membrane disrupts its function and causes it to collapse and have range of cytotoxic radicals. The most serious one is byproducts aldehydes. They may also react with transition metals like iron or copper to form stable aldehyde such as malodialdehyde for example, which will damage the cell membrane. Because hemoglobin constitutes the largest iron store in the body, it is speculated to be a potential source of redox activity iron, which can catalyze the formation of free radicals that might be damaging to the joint [8].

Antioxidant defense mechanisms involve both enzymatic and non-enzymattic strategies [9].

The aetiological factors of TMJ disorders are as follows: systemic diseases (rheumatoid arthritis, psoriasis, pseudogout, ankylosing spondylitis, etc.), secondary inflammatory component from the neighbouring regions (otitis, maxillary sinusitis, tonsillitis), trauma (chronical), prevalence of dental arch defects e.g. missing of molar teeth [10], malocclusion, endocrinological disturbances, odontogenic infections (impacted third molars) [11].

Presences of specific bacterial organisms such as Staph. aureus, Strep. mitis, M. fermentas, Actinobacillus actinomycetemcomitans in the synovial fluid have been found [12]. Serum antibodies against Chlamydia species in patients with mono arthritis of the TMJ have also been reported [13].

To realize this aim, the preparation of bio-active scaffolds which mimic the complexity of bone structure from the macro- to the molecular scale and which enable an activation of cell adhesion and proliferation under the stimulus of natural bio-adhesion in vitro through investigation of dentin bond strength as a model system is done.

Chitosan (CS), obtained through the alkaline deacetylation of chitin, is a copolymer of N-acetyl-D-glucosamine and D-glucosamine [14]. CS stands out by a unique combination of favorable biological properties such as nontoxicity, biocompatibility and biodegradability, along with mucoadhesive, bacteriostatic, and wound-healing properties [15]-[18].

In this work, Bioactive-functionalized interpenetrating network (IPNs) hydrogel (BIOF-INPs) were prepared and investigated in vitro for the free radical detection/defense, therapeutic release, ability to re-mineralize surface of the dentin after application of these bio-inspired materials using a biologically inspired mineralization process in vitro as well as investigating antimicrobial properties of the BIOF-INPs against S. aureous. The aim of this investigation was to evaluate the suitability and flexibility of the designer materials to act as an "in vitro" probe to gain insights into molecular origin of TMD and associated disorders.

\section{Preparation of FeEDTA-BIOF-INPs (BIOF-INPs)}

The novel prepared by dispersion of therapeutic agent such as naproxen, ibuprofen or aspirin (powder 0.4 gm) in glycerol $(5 \% \mathrm{w} / \mathrm{w})$ using a mortar and a pestle. FeEDTA $(5 \% \mathrm{w} / \mathrm{w})$ was added to the mixture and mortar and pestle used to achieve the uniform mixture. Ten milliliters of glacial acetic acid $(1 \% \mathrm{w} / \mathrm{w})$ was then added with continuous mixing and finally chitosan polymer was spread on the surface of the dispersion and mixed well to form the required gel and left for 24 hours. The strength of the prepared gel (10 gm) is $0.4 \mathrm{~g}$ therapeutic agentin each gram of the base. The summary of the newly prepared materials was presented in Table 1.

\subsection{In Vitro Study of Therapeutic Agents Release Profile}

The in vitro release of the therapeutic agents were evaluated using previously reported protocol [19]-[25]. Samples $(2 \mathrm{ml})$ were collected at a regular time intervals and were analyzed spectrophotometrically by U.V. Spectrophotometer (Cintra 5, GBC Scientific equipment, Australia) at $\lambda_{\max } 275 \mathrm{~nm}, \lambda_{\max }$ (ibuprofen) $264 \mathrm{~nm}$ and $\lambda_{\max }$ 
Table 1. Gel formulation prepared in the study.

\begin{tabular}{cccccccc}
\hline Gel formulation & & $\begin{array}{c}\text { Chitosan: FeEDTA } \\
\text { VitC 5:1 (w/w\%) }\end{array}$ & $\begin{array}{c}\text { Aspirin } \\
\text { (w/w\%) }\end{array}$ & $\begin{array}{c}\text { Ibuprofen } \\
\text { (w/w\%) }\end{array}$ & $\begin{array}{c}\text { Naproxen } \\
\text { (w/w\%) }\end{array}$ & $\begin{array}{c}\text { Tetracycline } \\
\text { (w/w\%) }\end{array}$ & pH \\
\hline Chitosan-VitC (5:1) & Gel-1 & 5 & 0 & 0 & 0 & 0 & 6.84 \\
Chitosan-VitC-A1 & Gel-2 & 5 & 1 & 0 & 0 & 0 & 6.92 \\
Chitosan-VitC-N1 & Gel-3 & 5 & 0 & 0 & 1 & 0 & 6.75 \\
Chitosan-VitC-I1 & Gel-4 & 5 & 0 & 1 & 0 & 0 & 6.56 \\
Chitosan-VitC-T1 & Gel-5 & 5 & 0 & 0 & 0 & 1 & 6.26 \\
\hline
\end{tabular}

VitC is vitamin $\mathrm{C}$ additive, FeEDTAas $\mathrm{Fe}^{2+} / \mathrm{Fe}^{3+}$ additive, $\mathrm{A}$ is aspirin additive, $\mathrm{N}$ is the naproxen, I-ibuprofen. Hydrogels containing chitosan:Vitamin C (5:1) (5\%) are synthesized and characterized.

(naproxen) $260 \mathrm{~nm}$ [19]-[25]. Each sample was replaced by the same volume of phosphate buffer $\mathrm{pH} 6.8$ to maintain its constant volume and sink condition [19]-[25].

\subsection{Bioadhesive Investigation}

Bioadhesion studies were doneusing Chatillon apparatus for force measurement using previously reported protocol that has been published in detail [19]-[25]. This method determines the maximum force and work needed to separate two surfaces in intimate contact [19]-[25]. The strength was recorded as a function of the displacement, which allowed to determine the maximal detachment force, $\mathrm{F}_{\max }$, and the work of adhesion, $\mathrm{W}$, which was calculated from the area under the strength-displacement curve.

\subsection{Microbiological Investigations}

A type strain of Staphylococcus aureus (ATCC 12600), obtained from the American Type Culture Collection (Manassas, USA) was used as test bacterium for estimating the antibacterial activity of the hydrogels. The antibacterial activity of the prepared tetracycline/antioxidant chitosan hydrogels were tested using the standard Kirby-Bauer agar disc diffusion method (Bauer et al.). Five to $6 \mathrm{~mm}$ deep Muller-Hinton agar (Oxoid, Basingstoke, UK) plates were inoculated by streaking a standardized inoculum suspension that match a 0.5 McFarland standard and containing $10^{7}-10^{8}$ colony forming units/ml with a throat cotton swab. For each test sample $500 \mu \mathrm{g}$ of hydrogel was applied to a $6 \mathrm{~mm}$ diameter paper disc. The paper discs were placed on the inoculated MullerHinton agar medium and incubated at $37^{\circ} \mathrm{C}$ for 24 hours. The diameter of the zones of growth inhibition was measured with a caliper. Each measurement was done in triplicate and the testing of each sample was repeated 3 times. The antibacterial efficacy of the prepared gels were compared to antibiotic sensitivity discs (Mast Laboratories, Merseyside UL) containing $30 \mu \mathrm{g}$ of tetracycline per disc.

\section{Results and Discussion}

\subsection{The Characterization of FeEDTABIOF-INPs (Gel 1-4)}

The SEM images were obtained to characterize the microstructure of the freeze-dried naproxen composite gels and are presented in Figure 1. It could be seen that the hydrogels have a channel-like surface with an extremely porous structure. While the hydrogels have a similar surface appearance, the distribution and the size of the pores are different. The detailed investigation into the correlation of the pore sizes versus the concentration of the cross-linking moieties incorporated into the gel are currently under investigation in our laboratory and will be reported in the due course. The "skin" of the gels can be seen, and the collapse of the surface pores may be due to freeze-drying process.

\subsection{Bio-adhesion in Vitro Model}

Higher adhesiveness of the gels is desired to maintain an intimate contact with skin or tooth structure and results are summarized in Table 2. Chitosan hydrogels showed the highest adhesive force and the work of adhesion this can be expected because of the well known intrinsic bio-adhesive properties of chitosan [26] [27]. The adequate 


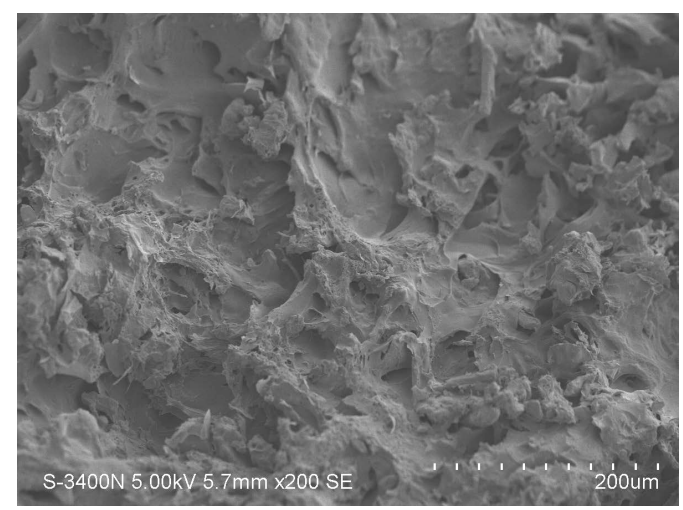

FeEDTA/Chit/Vitamin C

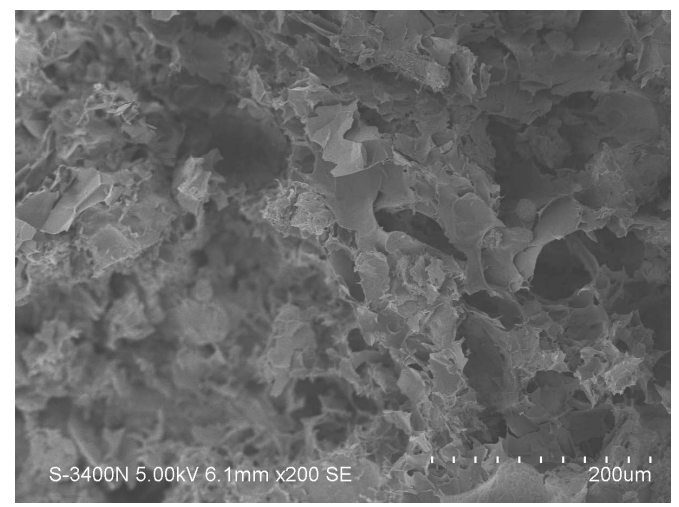

FeEDTA/Chit/VitC/Nap

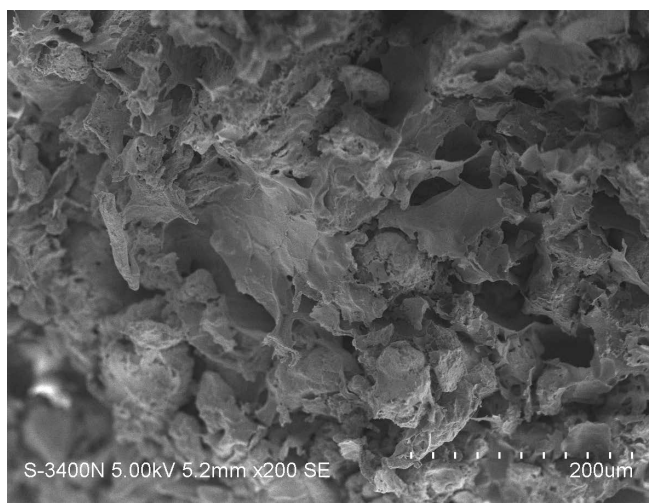

FeEDTA/Chit/VitC/lb

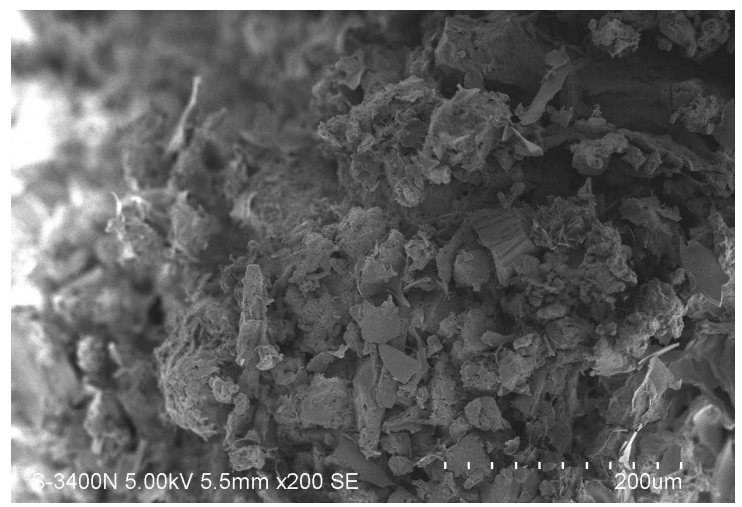

FeEDTA/Chit/VitC/Asp

Figure 1. SEM photographs of interior morphology of the selected gels under investigation for (a) Gel-1, (b) Gel-2, (c) Gel-3, and (d) Gel-4.

Table 2. Bioadhesion testing in vitro.

\begin{tabular}{ccc}
\hline Hydrogel & Adhesive Force $(\mathrm{N}) \pm \mathrm{SD}$ (Dentin) & Work of Adhesion $(\mathrm{Ncm}) \pm \mathrm{SD}$ (Dentin) \\
\hline Gel-1 & $1.91 \pm 0.35$ & $7.92 \pm 0.34$ \\
Gel-2 & $1.79 \pm 0.44$ & $8.49 \pm 0.42$ \\
Gel-3 & $1.89 \pm 0.60$ & $6.94 \pm 0.29$ \\
Gel-4 & $1.97 \pm 0.35$ & $7.35 \pm 0.34$ \\
\hline
\end{tabular}

The presented values are an average $(n=5)$.

water absorption capacity together with the cationic nature of the chitosan containing IPN promotes binding to the surface of dentin structure. Further investigations are currently on the way in our laboratory to establish the correlation between adhesive force and the skin surfaces and results will be reported elsewhere.

The correlation between the force and work of adhesion is noticeable for all. Further experiments are to be conducted on the skin samples to evaluate the bio-adhesive capacity of the designer hydrogels.

Interestingly the surface of the dentin surface has been significantly influenced by the treatment with Gels 1-4, confirming previously reported unique properties of the chitosan to promote formation of the hydroxyapatite crystals as well as prevent demineralization of enamel and dentin which has been reported previously [28] and therefor supports the initial hypothesis of the model system being suitable to investigate molecular mechanism of TMD in vitro using dentin as a prototype of fibrous articular disc model (Figure 2).

Once dentine, which represents our model materials, is subjected to the biochemically induced oxidative free radical stress, demineralisation of the dentine matrix initiates mineral loss from the extra-fibrillar as well as intra-fibrillar compartments of type I collagen fibrils [29] [30]. The loss of minerals from the intra-fibrillar com- 


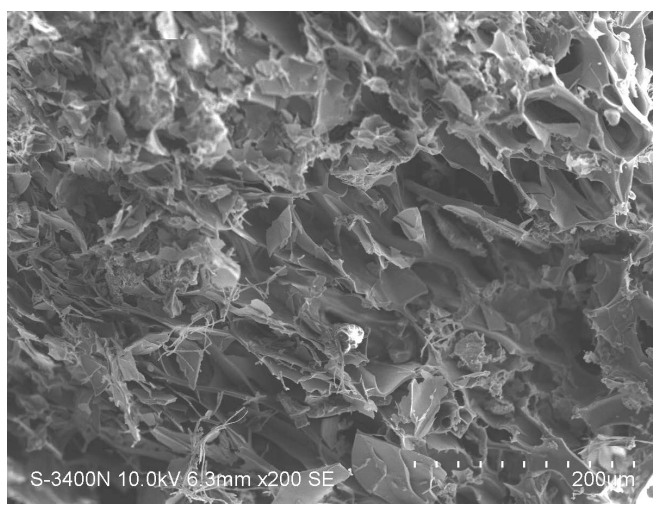

Surface exposed to Gel 1

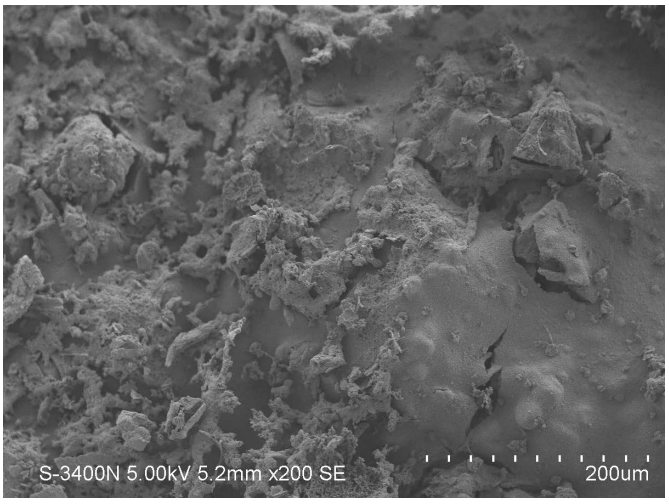

Surface exposed to Gel 3

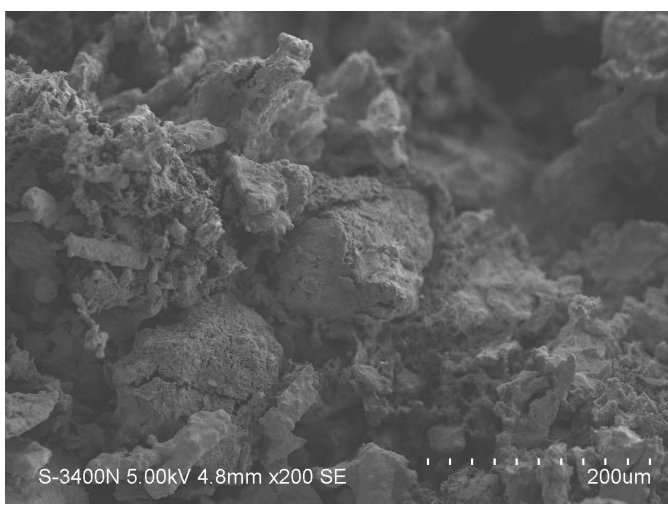

Surface exposed to Gel 2

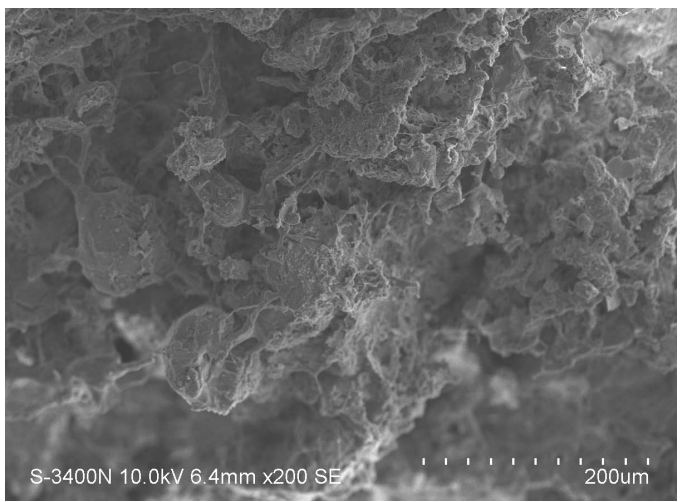

Surface exposed to Gel 4

(a)

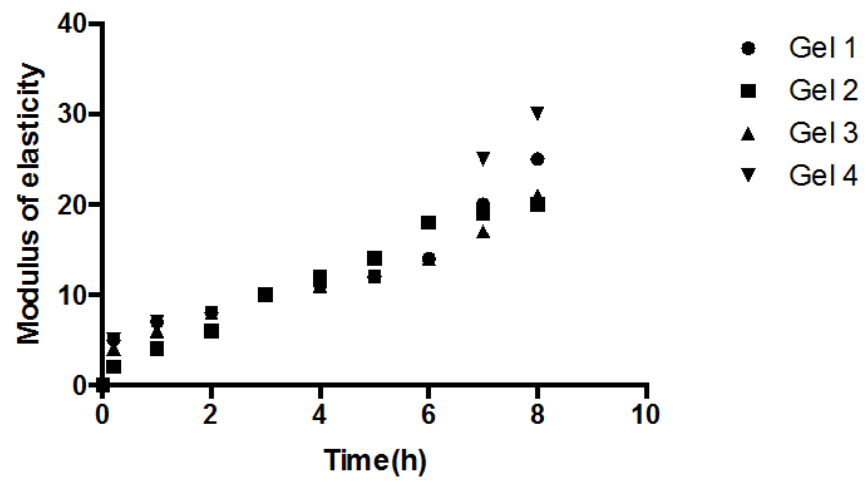

(b)

Figure 2. (a) SEM images of the dentin surface exposed to BIOF-INPs 1-4 for 3 weeks; (b) Modulus of elasticity and reaction to the hydrogels.

partments results in lower mechanical properties of dentine [30]. With the dissolution of minerals, the exposed collagen is subjected to various exogenous as well as host-derived collagenolytic enzymes, resulting in enzymemediated collagen breakdown [31]. The regeneration treatment of affected dentine is challenging due to the loss of the integrity of the dentine collagen matrix [32] [33]. Hence, modifying the dentine matrix by strengthening the collagen fibrillar matrix appears to be a rational approach for withstanding mechanical forces, resisting degradation by proteases as well to reducing collagen-bound proteoglycans [24] [34]. Cross-linking of the collagen matrix provides an effective solution for preserving and repairing the de-mineralised dentine structure. This study has shown that newly developed hydrogels are capable to improve the modulus of elasticity of de-mineraliseddentine, the detailed investigation of the potential mechanism is currently on the way in our laboratory. 


\subsection{In Vitro Release of Therapeutic Agents from FeEDTA Bioactive-Functionalized Interpenetrating Network (IPNs) Hydrogel (BIOF-INPs)}

The in vitro release of therapeutic agents from $\mathrm{Fe}^{2+}$ (BIOF-INPs) was carried out using USP dissolution apparatus type I. As the regression analysis of the obtained results for two kinetic models including zero order and Higushi's model showed that Higushi's model gave the highest value of $r^{2}$ with significant difference $(p<0.05)$ [24]. Higushi's model, where the cumulative amount of the released drug per unit area is proportional to the square root of time, is more suitable model to describe the release kinetics of from the gel preparations examined in the present study. The release of therapeutic agents from the FeEDTA-BIOF-INPs was studied and gels containing corresponding antioxidant, as shown in Figure 3.

The controlled release of the therapeutic agents under investigation depends on $\mathrm{pH}$ value of the media, with slower release kinetics at the higher $\mathrm{pH}$ conditions, which was attributed to amino groups of chitosan protonation, resulting in a soluble and positively charged polysaccharide leading to faster swelling in the acidic media. The plot reveals that there are 2 stages for the release of the therapeutic agents in all Gels. The first stage of release was initially rapid (burst release), which maybe result from the rapid diffusion of therapeutic agents form the FeEDTABIOF-INPs and the initial swelling. The second stage of release of the all investigated therapeutic agents demonstrates a slow, controlled release. The burst release has an advantage in reaching a biologically relevant concentration much faster, where as slow release will control the sustainable concentration of therapeutic agents such as naproxen, ibuprofen or aspirin in plasma, for example for a prolonged period of time.

\subsection{Free Radical Defense Capability of the Prepared Hydrogels}

The amount of uncontrolled ROS is the main cause of the inability of healing process to continue and therefor it would be ideal to utilize the antioxidant capacity of the "designer hydrogels" to detect and able to "fight the free radical excess" have been assessed using previously described model using well-established that HO radical can be generated from a reaction known as the biologic Fenton reaction and this reaction requires the presence of $\mathrm{H}_{2} \mathrm{O}_{2}$.

Bovine serum albumin (BSA), a completely water-soluble protein, was polymerized by hydroxyl radicals generated by the Fenton reaction system of $\mathrm{Fe}^{2+} /$ EDTA/ $\mathrm{H}_{2} \mathrm{O}_{2}$ /ascorbate [19]-[25]. As a result, the protein loses its water-solubility and the polymerized product precipitates. The decrease in the concentration of the water-soluble protein can easily be detected.

Therefore we adopted the method for recording changes in water solubility of the model protein bovine serum albumin (BSA) exposed to free radicals generated by an inorganic chemical system. As clearly demonstrated by the Figure 4, upon exposure to standard $\mathrm{H}_{2} \mathrm{O}_{2}$ in the form of FeEDTA BIO-INPs (build in free radical material defense material) and $\mathrm{H}_{2} \mathrm{O}_{2}$ as a base line determinate free radical generation under "prototype in-vitro free radical damage", upon incorporation of the chitosan substituted hydrogels, the build in antioxidant capacity and therefor free radical defense of the in-vitro model has been activated and are of significant value to take notice. This model represents the practical approach of in-situ monitoring and test the amount of free radical production and synergistic antioxidant defense of the system. Further investigations and fine-tuning of the system are currently on the way in our laboratory.

The in-depth investigation of interaction between free radical "in-situ" production and functional chitosan biomaterials are currently on the way in our laboratory and will be reported in due course.

\subsection{Insight into Microbiological Investigations}

Paper discs impregnated with the chitosan hydrogels without tetracycline gave no inhibition zones. However all the test samples containing tetracycline give inhibition zones larger than the clinical breakpoint inhibition zone diameters (European Committee on Antimicrobial Susceptibility Testing, Basel, Switzerland) for S. aureus sensitivity for tetracycline. Using the students T test, no statistically significant difference $(p<0.05)$ between the averages of the inhibition zone diameters for all the samples were found (Table 3).

In the present study, tetracycline was selected because of its wide application, both locally and systemically, in TMJ potential treatment. A commercially available standard bacterial strain was selected to investigate whether the preliminary goal of preserving the activity of antibiotics, after incorporation into preparations, can be obtained. 


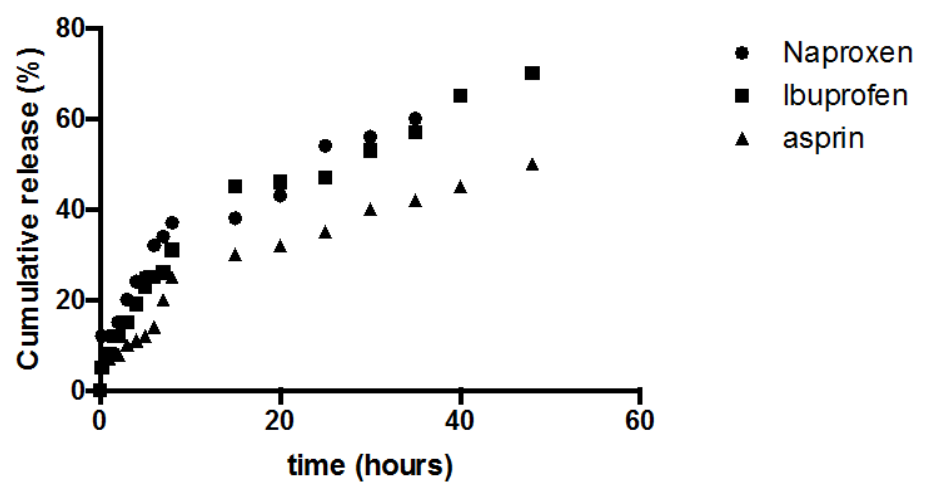

Figure 3. Cumulative release (\%) of naproxen, ibuprofen and aspirin from 5\% w/w FeEDTA-BIOF-INPs in phosphate buffer pH 6.8.

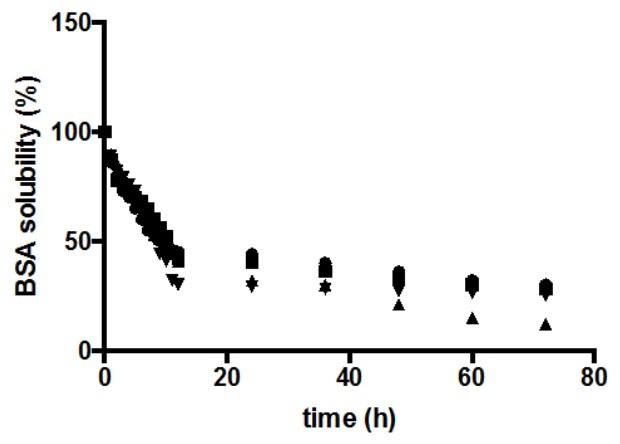

- $\mathrm{H}_{2} \mathrm{O}_{2}$ exposure

- Ibuprofen

- asprin

v naproxen

Figure 4. Plot of changes in water solubility of the model protein bovine serum albumin (BSA) over a time (72 hours) exposed to free radicals detected by a FeEDTABIOF-INPs and produced $\mathrm{H}_{2} \mathrm{O}_{2}$ /ascorbate system as a source of free radicals.

Table 3. (Tetracycline) tested for antibacterial activity against Staphylococcus aureus NCTC 12600.

Tetracycline inhibition zone diameters

Sample no. (n=10) Tetracycline disc $(30 \mu \mathrm{g}) \mathrm{Ch} / \mathrm{VitC}$ Ch/VitC/FeEDTA Ch/VitC/FeEDTA/T Ch/VitC/FeEDTA/T/HA Ch/VitC/T/HA

Average \pm standard deviation

$26.44 \pm 0.28$

0

0

$23.67 \pm 0.55$

$20.09 \pm 1.63$

$22.80 \pm 0.64$

Clinical breakpoints for tetracycline: $30 \mu \mathrm{g} /$ disc $>22 \mathrm{~mm}$ is sensitive and $<19 \mathrm{~mm}$ is resistant.

Our results showed that the cross-linked chitosan sponges were able to deliver active antibiotic for up to 5 days. Chitosan hydrogel scaffolds were designed in this study as carriers for antibiotics and showed a steady release of the medication. Three-dimensional chitosan matrices have been shown to be excellent tissue engineering scaffolds for cell attachment and growth. Chitosan has a scalloped structure and has been used in tissue engineering to culture hepatocytes, fibroblasts and cartilage cells because of its ability to promote cell attachment and growth [19]-[25].

In our investigation, chitosan was selected as the carrier for tetracycline, mainly because it can both carry and deliver the medication, but also because it has other useful bioactivities such as antioxidant and anti-inflammatory properties [19]-[25].

\section{Conclusion}

We developed and tested functional dual-action restorative materials based on FeEDTA-BIOF-INPs "build in free radical defense and detector", chitosan (antioxidant containing material as well as hydroxyapatite formation stimulant), naproxen, ibuprofen (non-steroidal anti-inflammatory medication), aspirin (pain relief medication 
and free radical scavengers), tetracycline (model antibiotic compound) and combinations thereof. We quantified the effects of functional designer biomaterials on the dentin bond strength of a composite and evaluate the capability of newly designed hydrogels to play an integral role of "build in free radical defense mechanism by using BSA solubility as a "molecular prototype of the site of free radical attack in vitro". The newly developed hydrogels also increase modulus of elasticity of the demineralized dentin. Therefore, the model functional multi-dimensional restorative repair materials (BIOF-INP) with the build in free radical defense mechanism act as a "proof of concept" for the potential molecular probe towards understanding TMD mechanism in vitro and potential development of the efficient treatment in vivo in the future.

\section{Acknowledgements}

VTP would like to thank VTPCHEM PTY LTD for financial support.

\section{References}

[1] McCullen, S.D., Chow, A.G. and Stevens, M.M. (2011) In Vivo Tissue Engineering of Musculoskeletal Tissues. Current Opinion in Biotechnology, 22, 715-720. http://dx.doi.org/10.1016/j.copbio.2011.05.001

[2] Panseri, S., Russo, A., Cunha, C., Bondi, A., Di Martino, A., Patella, S. and Kon, E. (2012) Osteochondral Tissue Engineering Approaches for Articular Cartilage and Subchondral Bone Regeneration. Knee Surgery, Sports Traumatology, Arthroscopy, 20, 1182-1191. http://dx.doi.org/10.1007/s00167-011-1655-1

[3] Milam, S.B. and Schmitz, J.P. (1995) Molecular Biology of Temporo-Mandibular Joint Disorders: Proposed Mechanisms of Disease. Journal of Oral and Maxillofacial Surgery, 53, 1448-1454. http://dx.doi.org/10.1016/0278-2391(95)90675-4

[4] Chan, P.H. (2001) Reactive Oxygen Radicals in Signaling and Damage in the Ischemic Brain. Journal of Cerebral Blood Flow \& Metabolism, 21, 2-14. http://dx.doi.org/10.1097/00004647-200101000-00002

[5] Irmak, MK., Fadıllığlu, E. and Güleç, M. (2002) Effects of Electromagnetic Radiation from a Cellular Telephone on the Oxidant and Antioxidant Levels in Rabbits. Cell Biochemistry and Function, 20, 279-283. http://dx.doi.org/10.1002/cbf.976

[6] Yaser, M.M., Randa, M.M. and Belacy, A. (2001) Effects of Acute Exposure to the Radiofrequency Fields of Cellular Phones on Plasma Lipid Peroxide and Antioxidase Activities in Human Erythrocytes. Journal of Pharmaceutical and Biomedical Analysis, 26, 605-608. http://dx.doi.org/10.1016/S0731-7085(01)00492-7

[7] Pompella, A. (1997) Biochemistry and Histochemistry of Oxidant Stress and Lipid Peroxidation. International Journal for Vitamin and Nutrition Research, 67, 289-297.

[8] Sumii, H., Inoue, H., Onoue, J., Mori, A., Oda, T. and Tsubokura, T. (1996) Superoxide Dismutase Activity in Arthropathy: Its Role and Measurement in the Joints. Hiroshima Journal of Medical Sciences, 45, 51-55.

[9] Arinzeh, T.L., Tran, T., McAlary, J. and Daculsi, G. (2005) A Comparative Study of Biphasic Calcium Phosphate Ceramics for Human Mesenchymal Stem-Cell-Induced Bone Formation. Biomaterials, 26, 3631-3638. http://dx.doi.org/10.1016/j.biomaterials.2004.09.035

[10] Holmlund, A.B. and Axelsson S. (1996) Temporomandibular Arthropathy: Correlation between Clinical Signs and Symptoms and Arthroscopic Findings. International Journal of Oral \& Maxillofacial Surgery, 25, 266-271. http://dx.doi.org/10.1016/S0901-5027(96)80024-5

[11] Emshoff, R. (2005) Clinical Factors Affecting the Outcome of Arthrocentesis and Hydraulic Distension of the Temporomandibular Joint. Oral Surgery, Oral Medicine, Oral Pathology, Oral Radiology, and Endodontology, 100, 409-414. http://dx.doi.org/10.1016/j.tripleo.2004.12.024

[12] Voog, Ü., Alstergren, P., Eliasson, S., Leibur, E., Kallikorm, R. and Kopp, S. (2003) Inflammatory Mediators and Radiographic Changes in Temporomandibular Joints in Patients with Rheumatoid Arthritis. Acta Odontologica Scandinavica, 61, 57-64.

[13] Kim, S.J., Park, Y.H., Hong, S.P., Cho, B.O., Park, J.W. and Kim, S.G. (2003) The Presence of Bacteria in the Synovial Fluid of the Temporomandibular Joint and Clinical Significance: Preliminary Study. Journal of Oral and Maxillofacial Surgery, 61, 1156-1161. http://dx.doi.org/10.1016/S0278-2391(03)00674-8

[14] Paegle, D.I., Holmlund, A.B., öStlund, M.R. and Grillner, L. (2004) The Occurrence of Antibodies against Chlamydia Species in Patients with Monoarthritis and Chronic Closed Lock of the Temporomandibular Joint. Journal of Oral and Maxillofacial Surgery, 62, 435-439. http://dx.doi.org/10.1016/j.joms.2003.08.020

[15] Leibur, E., Jagur, O., Müürsepp, P., Veede, L. and Voog-Oras, Ü. (2010) Long-Term Evaluation of Arthroscopic Surgery with Lysis and Lavage of Temporomandibular Disorders. Journal of Cranio-Maxillofacial Surgery, 38, 615-620. http://dx.doi.org/10.1016/j.jcms.2010.02.003 
[16] Alstergren, P., Kopp, S. and Theordosson, E. (2003) Synovial Fluid Sampling from the Temporomandibular Joint: Sample Quality Criteria and Levels of Interleukin-1 Beta and Serotonin. Acta Odontologica Scandinavica, 57, $278-282$.

[17] Kamada, A., Kakudo, K., Arika, T., Okazaki, J., Kano, M. and Sakaki, T. (2000) Assay of Synovial MMP-3 in Temporomandibular Joint Diseases. Journal of Cranio-Maxillofacial Surgery, 28, 247-248.

[18] Alstergren, P. and Kopp, S. (1997) Pain and Synovial Fluid Concentration in Arthritic Temporomandibular Joints. Pain, 72, 137-143.

[19] Perchyonok, V., Zhang, S. and Oberholzer, T. (2011) Towards Development of Novel Chitosan Based Drug Delivery Prototypes: Devices for Targeted Delivery Drug Therapy at the Molecular Level in Aqueous Media. Current Organic Chemistry, 16, 2437-2439.

[20] Perchyonok, V.T., Reher, V., Zhang, S., Grobler, S.R., Oberholzer, T.G. and Massey, W. (2014) Insights and Relative Effect of Aspirin, Naproxen and Ibuprofen Containing Hydrogels: From Design to Performance as a Functional Dual Capacity Restorative Material and Build in Free Radical Defense: In-Vitro Studies. Open Journal of Stomatology, 4, 73-83. http://dx.doi.org/10.4236/ojst.2014.42013

[21] Perchyonok, V., Zhang, S. and Oberholzer, T. (2012) Novel Melatonin-Chitosan Hydrogels as Suitable Oral Bio-Drug Delivery Systems to Fight Oral Mucositis: Synergy of Antioxidants and Bioactives in Action. Current Organic Chemistry, 16, 2430-2436.

[22] Perchyonok, V.T., Zhang, S., Grobler, S.R., Oberholzer, T.G. and Massey, W. (2014) Insights into and Relative Effect of Chitosan-Krill Oil, Chitosan-H-Aspirin, Chitosan-H-Krill Oil-Nystatin and Chitosan-H-Krill Oil-Aspirin-Nystatin on Dentin Bond Strength and Functional Drug Delivery Capacity: In-Vitro Studies. European Journal of General Dentistry, 3, 57-65.

[23] Perchyonok, V.T., Zhang, S., Basson, N.J., Grobler, S.R., Oberholzer, T.G. and Massey, W. (2014) Insights into Functional Erythromycin/Antioxidant Containing Chitosan Hydrogels as Potential Bio-Active Restorative Materials: Structure, Function and Antimicrobial Activity. Advanced Techniques in Biology and Medicine, 2, 116.

[24] Perchyonok, V.T., Zhang, S., Basson, N.J., Grobler, S.R., Oberholzer, T.G. and Massey, W. (2014) Insights into Functional Tetracycline/Antioxidant Containing Chitosan Hydrogels as Potential Bio-Active Restorative Materials: Structure, Function and Antimicrobial Activity. Open Journal of Stomatology, 4, 99-108. http://dx.doi.org/10.4236/ojst.2014.43016

[25] Perchyonok, V.T., Zhang, S., Grobler, S.R. and Oberholzer, T.G. (2013) Insights into and Relative Effect of Chitosan-H, Chitosan-H-Propolis, Chitosan-H-Propolis-Nystatin and Chitosan-H-Nystatin on Dentine Bond Strength. European Journal of Dentistry, 7, 412-418.

[26] Tampieri, A., Celotti, G., Landi, E., Sandri, M., Roveri, N. and Falini, G. (2003) Biologically Inspired Synthesis of Bone-Like Composite: Self-Assembled Collagen Fibers/Hydroxyapatite Nanocrystals. Journal of Biomedical Materials Research Part A, 67, 618-625.

[27] Tampieri, A., Sandri, M., Landi, E., Pressato, D., Francioli, S., Quarto, R. and Martin, I. (2008) Design of Graded Biomimetic Osteochondral Composite Scaffolds. Biomaterials, 29, 3539-3546. http://dx.doi.org/10.1016/j.biomaterials.2008.05.008

[28] Zhao, H., Wang, G., Hu, S., Cui, J., Ren, N., Liu, D., Liu, H., Cao, C., Wang, J. and Wang, Z. (2011) In Vitro Biomimetic Construction of Hydroxyapatite-Porcine Acellular Dermal Matrix Composite Scaffold for MC3T3-E1 Preosteoblast Culture. Tissue Engineering Part A, 17, 765-776. http://dx.doi.org/10.1089/ten.tea.2010.0196

[29] Bernhardt, A., Lode, A., Boxberger, S., Pompe, W. and Gelinsky, M. (2008) Mineralised Collagen-An Artificial, Extracellular Bone Matrix-Improves Osteogenic Differentiation of Bone Marrow Stromal Cells. Journal of Materials Science: Materials in Medicine, 19, 269-275. http://dx.doi.org/10.1007/s10856-006-0059-0

[30] Domaschke, H., Gelinsky, M., Burmeister, B., Fleig, R., Hanke, T., Reinstorf, A., Pompe, W. and Rösen-Wolff, A. (2006) In Vitro Ossification and Remodeling of Mineralized Collagen I Scaffolds. Tissue Engineering, 12, 949-958.

[31] Hou, R., Zhang, G., Du, G., Zhan, D., Cong, Y., Cheng, Y. and Fu, J. (2013) Magnetic Nanohydroxyapatite/PVA Composite Hydrogels for Promoted Osteoblast Adhesion and Proliferation. Colloids and Surfaces B: Biointerfaces, 103, 318-325. http://dx.doi.org/10.1016/j.colsurfb.2012.10.067

[32] Mertens, M.E., Hermann, A., Bühren, A., Olde-Damink, L., Möckel, D., Gremse, F., Ehling, J., Kiessling, F. and Lammers, T. (2014) Iron Oxide-Labeled Collagen Scaffolds for Non-Invasive MR Imaging in Tissue Engineering. Advanced Functional Materials, 24, 754-762.

[33] Almeida, J.P.M., Chen, A.L., Foster, A. and Drezek, R. (2011) In Vivo Biodistribution of Nanoparticles. Nanomedicine, 6, 815-835. http://dx.doi.org/10.2217/nnm.11.79

[34] Mahmoudi, M., Hofmann, H., Rothen-Rutishauser, B. and Petri-Fink, A. (2012) Assessing the in Vitro and in Vivo Toxicity of Super-Paramagnetic Iron Oxide Nanoparticles. Chemical Reviews, 112, 2323-2338. http://dx.doi.org/10.1021/cr2002596 